\title{
Translocations dans les syndromes lympho- et myéloprolifératifs : un ordre dans le désordre
}

\section{Les remaniements} chromosomiques semblent extrêmement fréquents dans les cancers humains, et notamment dans ceux des lignées hématopoiétiques. $A$ travers la diversité des situations, existe-t-il un fil conducteur, en quelque sorte une "systématique " des réarrangements conduisant à la cancérisation d'une lignée donnée. Laurent Degos, et d'autres, pensent que la juxtaposition d'un oncogène et d'un gène spécialement actif dans la cellule cible du processus malin pourrait constituer cette "clé" permettant de comprendre le mécanisme de la cancérisation es données sur les anomalies chromosomiques dans les syndromes lympho-et myéloprolifératifs s'accroissent et il est difficile de s'y reconnaître. Cependant les translocations ne se font pas au hasard. Les cytogénéticiens retrouvent toujours la même bande sur tel ou tel chromosome impliquée dans un remaniement donné [1]. Autre argument apporté par les cytologistes, en collaboration avec les cytogénéticiens, une translocation particulière correspond à un type, voire à un sous-type de leucémie ou de lymphome [1]. Enfin, la très belle démonstration du lymphome de Burkitt [2] nous révèle qu'au cours de ces remaniements, il est fréquent que s'apparient deux gènes, l'un dit oncogène ( $c$-myc) et l'autre en pleine activité transcriptionnelle, probablement en raison de l'état de différenciation de la cellule, comme les gènes des immunoglobulines dans ce cas de tumeur atteignant le lymphocyte B. Le hasard n'existe pas, mais existe-t-il un ordre ?

"Les oncogènes cellulaires sont des gènes cellulaires qui interviennent dans le contrôle normal de la prolifération et de la croissance, voire de la différenciation et dont les modifications qualitatives ou des anomalies de l'expression entraînent l'acquisition du pouvoir transformant " [3].

Ainsi peut-on citer les relations entre un facteur de croissance (PDGF) et l'oncogène sis, certains récepteurs de facteurs de croissance et les oncogènes erb $B$, fms et neu, les $G$ protéines intervenant dans la transduction du message au travers de la membrane cellulaire et la famille des oncogènes ras, les protéines kinases intracellulaires et des oncogènes à activité tyrosine kinase tels que src et abl, ou à activité sérinethréonine kinase tels que mos, des protéines se liant à l'ADN et régulant l'activité des gènes et les oncogènes myc, myb, fos, $E_{1} A$, ou l'antigène $T$ de polyome.

Suivant le modèle du lymphome de Burkitt, peut-on alors dire que les gènes impliqués dans une translocation sont de deux catégories : des oncogènes intervenant dans le cycle cellulaire, et des gènes ayant une action spécifique dans la cellule cible concernée, probablement des gènes dont l'activité serait conditionnée par l'état de différenciation?

Heim et Mitelman [4] ont proposé une voie de recherche classant les points de cassure en deux catégories. Dans la première catégorie une même bande cytogénétique se trouve impliquée dans des états prolifératifs différents. Inver- 


\section{ABRÉVIATIONS}

ALL : Leucémie aiguë lymphoblastique ( $T A L L$ : lymphoblastique $T)$

ANLL : Leucémie aiguë non lymphoblastique $(=$ myéloblastique)

Burkitt : Lymphome ou leucémie (L3) de Burkitt

CLL : Leucémie lymphoïde chronique (TCLL : lymphoïde chronique $T$ )
CML : Leucémie myéloïde chronique

CMML : Leucémie myélomonocytaire chronique

FOLL-LS : Lymphome folliculaire Ig $H$ : Chaîne lourde des immunoglobulines

Ig $k$ : Chaîne légère kappa des immunoglobulines

Ig $\lambda$ : Chaîne légère lambda des immunoglobulines
Mal.His. : Histiocytose maligne MDS : Syndrome myélodysplasique Myélop. : Syndrome myéloprolifératif

M3 : Leucémie à promyélocyte (LAM 3)

M4 Eo : Leucémie myélomonocytaire aiguë avec éosinophiles

M5a : Leucémie monoblastique $T c R$ : Récepteur antigène $d u$ lymphocyte $T$

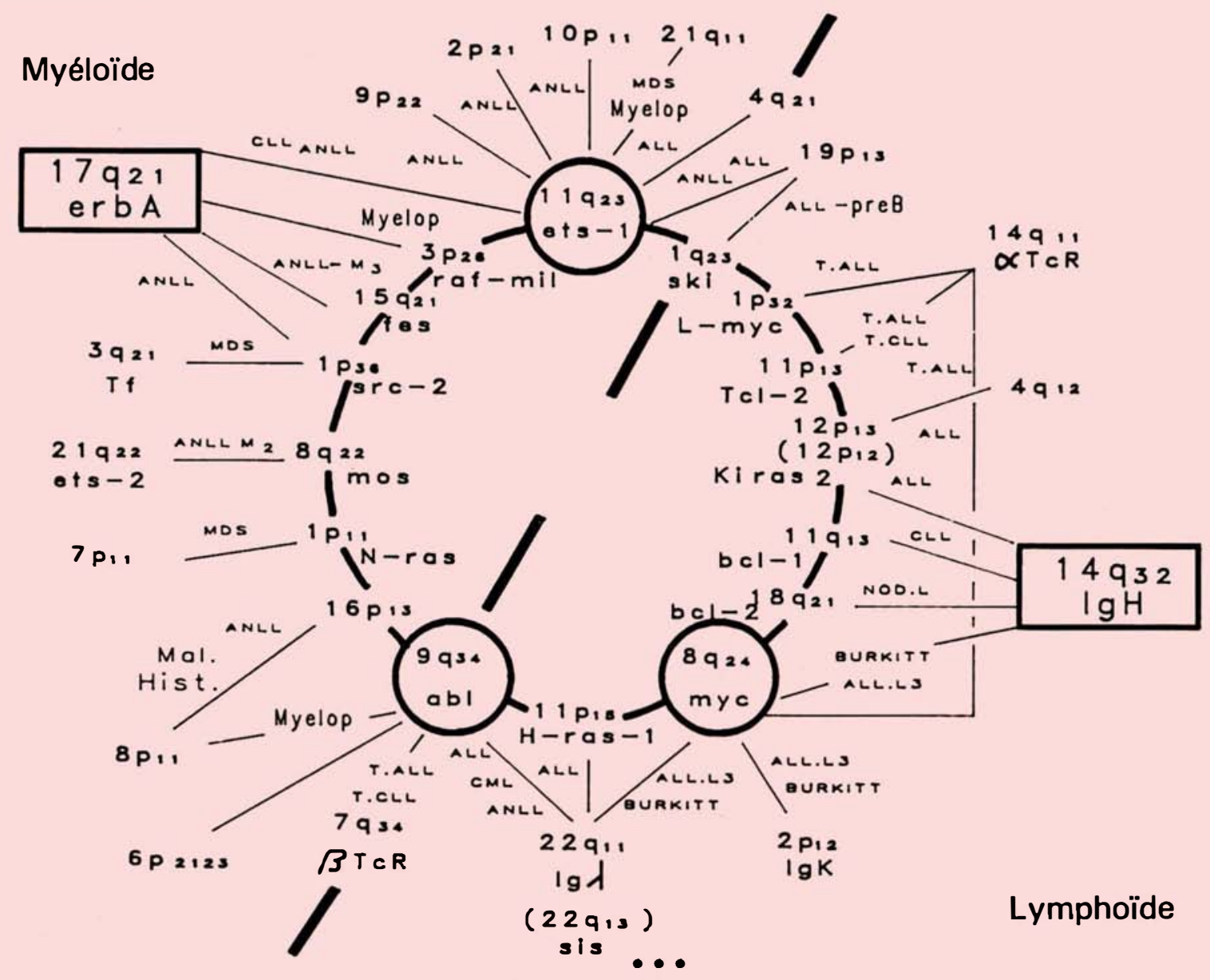

Figure 1. Translocation dans les proliférations lymphoïdes et myéloïdes. Les points de cassure de chaque translocation sont reliés par un trait. Par exemple, 8q24-14q32. Les oncogènes proches de ces points de cassure sont aussi indiqués. II existe pour chaque translocation un gène impliqué dans la croissance cellulaire (placé sur le cercle) associé à un gène de différenciation lymphoïde ou myéloïde.

$\mathrm{m} / \mathrm{s} n^{\circ} 3$ vol. 3, mars 87 
sement, dans la deuxième catégorie, certaines bandes se trouvent impliquées dans de nombreuses translocations au cours de proliférations touchant la même lignée. Nous avons regardé plus spécifiquement les proliférations lymphoïdes et myéloïdes. Par exemple dans la première catégorie, Heim et Mitelman citent la bande 11q23 qui est inscrite dans de nombreuses translocations (avec 9p22, 2p21, 10p11, 21q11, $4 q 21$ et 19 p13) tantôt lors de leucémies non lymphoblastiques pour les trois premiers cas, tantôt lors de myélodysplasie avec 21q11, tantôt lors de leucémies lymphoblastiques pour les deux derniers. Cette bande 11q23 pourrait contenir un des gènes constituant un chaînon commun du cycle cellulaire identique quelle que soit la lignée de la cellule. Autre exemple, 9q34 est inclus dans des translocations avec 22q11 (leucémie aiguë lymphoblastique, leucémie myéloïde chronique, leucémie aiguë non lymphoblastique) avec $7 q 34$ (leucémie aiguë lymphoblastique ou leucémie lymphoïde chronique $\mathrm{T}$ ) ou avec 8p11 (syndrome myéloprolifératif). Là encore, 9q34 pourrait être le siège d'un gène qui code pour une fonction cellulaire indépendante de la lignée, c'est-à-dire indépendante de la différenciation.

Pour la deuxième catégorie par exemple, la bande $14 \mathrm{q} 32$ associée à $12 \mathrm{p} 13,11 \mathrm{q} 13,18 \mathrm{q} 21$ ou $8 \mathrm{q} 24$ se retrouve dans des lymphoproliférations $\mathrm{B}$, chroniques ou aiguës. Autre exemple la bande 17q21 est associée à $11 \mathrm{q} 23,3 \mathrm{p} 26,15 \mathrm{q} 21$ ou 1 p36 dans des syndromes myéloprolifératifs. Ces bandes pourraient être la marque de la proximité de gènes spécifiques d'une lignée (ou d'un état de différenciation).

En faisant l'hypothèse que pour une translocation, lorsqu'un point de cassure entre dans une catégorie, l'autre point de cassure appartient à l'autre catégorie, et en essayant de prendre en considération toutes les translocations connues des proliférations lymphoïdes et myéloïdes, nous avons construit la figure 1 page précédente en plaçant au centre les gènes dits de prolifération, et en périphérie les gènes dits de différenciation. Inversement, Heim et Mitelman dans leur article avaient construit un cercle composé alternativement de gènes de prolifération et de gènes de différenciation, avec des branchements extérieurs ayant la même alternance, se servant de quelques exemples de points de cassure.

Si l'on peut décoder, c'est-à-dire savoir quel gène se trouve à proximité de la bande concernée [5], cette figure 1 apporte des enseignements et fait émettre des hypothèses. Ainsi, il existe une correspondance entre les gènes dits de prolifération et les gènes de croissance cellulaire (ras, myc, abl, src, mos, ski, fes) au centre de la figure sur lesquels se greffent en périphérie les partenaires des translocations représentant les gènes spécifiques de la lignée cellulaire (gènes d'immunoglobuline ou du récepteur $T$ pour la lignée lymphoïde par exemple).

Cependant la proximité d'un gène par rapport au point de cassure ne signifie pas qu'il soit remanié par la translocation. La biologie moléculaire a apporté certains exemples mettant en doute l'implication d'oncogènes voisins des points de cassure, ou démontrant que l'emplacement exact de la cassure était en fait différent d'un malade à un autre pouvant ainsi faire intervenir des gènes proches mais différents.

Que nous enseigne cette ordonnance des translocations dans les proliférations myéloïdes et lymphoïdes ? Peu d'oncogènes impliqués dans le cycle de croissance cellulaire (une quinzaine) sont présents mais leur nombre

\begin{tabular}{|c|c|c|}
\hline \multicolumn{3}{|c|}{ Tableau I } \\
\hline Anomalie chromosomique & État pathologique associé * & Gènes impliqués? \\
\hline 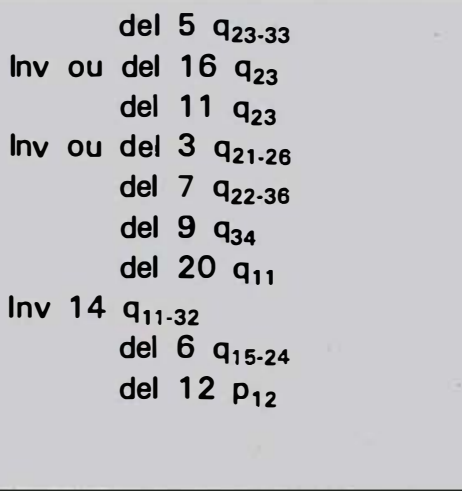 & $\begin{array}{l}\text { MDS } \\
\text { ANLL = M4 éosino } \\
\text { ANLL = M5 a } \\
\text { ANLL (M1) + thrombocytose } \\
\text { Leucémies secondaires } \\
\text { Myélop. } \\
\text { Myélop. } \\
\text { CLL } \\
\text { ALL } \\
\text { ALL } \\
\text { CMML } \\
\text { ANLL basophile }\end{array}$ & $\begin{array}{l}\text { GM CSF - fms (récepteur GM CSF) } \\
\text { Metallothionine } \\
\text { ets-1 } \\
\text { Transferrine - récepteur Tf } \\
\text { met } \\
\text { abl } \\
\text { src-1 } \\
\text { lg ; TCR } \alpha \\
\text { myb } \\
\text { ki-ras }\end{array}$ \\
\hline
\end{tabular}


limité est connu [3]. En revanche, peu de gènes propres à la lignée sont identifiés : pour les lymphoproliférations les trois gènes des immunoglobulines $(H, \varkappa, \lambda)$, les deux gènes du récepteur antigène des lymphocytes $\mathrm{T}$ (TcR alpha et bêta) ; d'autres restent inconnus, siégeant en 4q21, 4q12 et 19p13; pour les syndromes myéloprolifératifs erbA, transferrine, ets-2 ; erbA a une homologie avec les récepteurs nucléaires des stéroïdes et peut-être encore plus forte avec le récepteur de l'hormone thyroïdienne T3. L'homologie porte sur la partie codant pour l'attache de ce récepteur à l'ADN. Les androgènes stimulent la myélopoïèse, les glucocorticoïdes favorisent la chasse médullaire des cellules myéloïdes même peu matures. D'autres gènes proches de $7 \mathrm{p} 11$, 8p11, 9p22, 2p21, 10p11, 21q11 restent à découvrir. Ceci doit inciter à rechercher la nature de ces gènes en quête d'étiquette, correspondant à un marqueur de différenciation.

Certains gènes $(\mathrm{Tcl}, \mathrm{bcl})$ propres à une lignée (lymphocytes $T$, lymphocytes B) se trouvent placés par cette démarche sur la figure dans le cercle correspondant au cycle cellulaire. Ne seraient-ils pas les gènes des facteurs de croissance (ou de leurs récepteurs) spécifiques d'une lignée et donc jouant un rôle dans le cycle cellulaire mais de façon spécifique d'une lignée ? Ets-1 est un complexe avec, à proximité, des gènes codant pour des molécules d'adhésion cellulaire $(\mathrm{N}-\mathrm{Cam})$, un site fragile, le gène Thy1, et une chaîne du CD3 associée au récepteur antigène du lymphocyte $T$. Il existe cependant des exceptions à cette règle $(17 \mathrm{q} 21$, bande principalement impliquée dans les translocations des syndromes myéloprolifératifs, a été également retrouvée dans la leucémie lymphoïde chronique et, inversement, 19p13 lors de leucémies myéloblastiques). Cependant ces cas sont rares. Par ailleurs nous n'avons pas représenté la cassure de 6p21-23 associée dans des syndromes myéloprolifératifs à des remaniements se situant entre les

$m / s n^{\circ} 3$ ool. 3 , mars 87 bandes q12 et q25 du chromosome 1. La faible précision des sites de recombinaison ne permet en effet pas de les associer à des loci donnés. Enfin, certains points de cassure peuvent avoir une apparente similitude en cytogénétique, tout en faisant intervenir des gènes différents dans des états pathologiques variés (comme la région 22q11 ou 11q23).

Quelques gènes apparaissent comme les grands responsables des proliférations lymphoïdes et myéloïdes associées aux remaniements chromosomiques : $11 \mathrm{q} 23$ (ets-1), 8q24 (myc) et 9q34 (abl) pour le groupe des oncogènes, $14 \mathrm{q} 32$ ( $\mathrm{IgH}$ ) dans les proliférations lymphoïdes ou encore $17 q 21$ (erbA) dans les proliférations myéloïdes, représentent la très grande majorité des points de cassure des translocations répertoriées.

Inversement il y a des absents : par exemple, pour les lymphoproliférations $T$, les points de cassure $14 \mathrm{q} 11(\mathrm{TcR} \alpha)$ et $7 \mathrm{q} 34(\mathrm{TcR} \beta)$ sont bien retrouvés mais pas $7 \mathrm{p} 15$ qui correspond à la chaîne gamma.

Le schéma de la figure 1 est en fait simple. Il n'y a pas de croisement à l'intérieur du cercle. La dissociation myéloïde-lymphoïde est claire comme s'il existait des couples préférentiels entre oncogène du cycle de croissance et gène de différenciation. Dans la partie myéloïde, beaucoup d'oncogènes à activité protéine kinase se retrouvent (abl, src, fes, raf/mil, mos), alors que l'on remarque surtout myc dans le secteur lymphoïde. Seules les bandes $11 \mathrm{q} 23$ et $9 \mathrm{q} 34$ du cercle des oncogènes sont impliquées dans des remaniements divers dans l'une ou l'autre lignée. Mais ceci se compliquera certainement dans le futur. Cependant cette simplicité pourrait avoir une signification (existence de facteur, de récepteur, de voie de couplage, ou de phosphorylation spécifiques à chaque lignée ?).

Hormis les translocations, il existe aussi des délétions ou des inversions (Tableau I). Pour certaines d'entre elles le résultat est identique à celui d'une translocation mais intra-chromosomique. La délétion 3q21-26 met le gène de la transferrine (3q21) au contact de celui de son récepteur (3q26). La délétion 5q23-33 met à proximité l'oncogène fms (récepteur du GM-CSF) (5q33) avec le gène codant pour le facteur de croissance myéloïde GM-CSF (5q23). Ces délétions (ou inversions) impliquent pratiquement toujours une région où se situe un oncogène connu. Quelle est l'anomalie exacte ? une pseudo-translocation (intra-chromosomique) comme nous venons de le voir pour $3 q^{-}$et $5 q^{-}$, ou une délétion d'un "anti-oncogène ", gène codant pour une substance à effet antiprolifératif ?

Cette image ordonnée que schématise la figure 1 a certainement le défaut d'être simplificatrice mais aussi l'avantage de poser des questions, de chercher à aller plus loin, bien que l'on sache que les réarrangements réciproques (ou les délétions) ne sont pas nécessaires (et même pourraient ne pas être directement impliqués) dans l'origine de la malignité, mais peut-être seulement dans sa progression

\section{Laurent Degos \\ Professeur en hématologie clini- que à l'université Paris VII. Directeur de l'Unité Inserm U. 93, hôpital Saint-Louis, 2, place du Dr-A. -Fournier, 75475 Paris Cedex 10.}

\section{RÉFÉRENCES}

1. Berger R. Cytogénétique et cancer. médecine/sciences $1986 ; 2$ : 246-52.

2. Kaplan JC, Szajnert MF. Chromosome et cancer. médecine/sciences $1985 ; 1: 17-23$.

3. Kahn A. La saga des oncogènes. médecinelsciences $1985 ; 1$ : 10-1.

4. Heim S, Mitelman F. Proliferation-specific and differentiation-associated chromosomal break points in human neoplasia. An unifying model. Hereditas 1986 ; 104 : 307-12.

5. Stéhelin $D$. Les oncogènes cellulaires, clefs de la cancérogenèse. médecine/sciences 1985 ; 1 : 12-6. 